\title{
SOME REMARKS ON THE PLANAR KOUCHNIRENKO'S THEOREM
}

\author{
GERT-MARTIN GREUEL AND NGUYEN HONG DUC
}

\begin{abstract}
We consider different notions of non-degeneracy, as introduced by Kouchnirenko (NND), Wall (INND) and Beelen-Pellikaan (WNND) for plane curve singularities $\{f(x, y)=0\}$ and introduce the new notion of weighted homogeneous Newton non-degeneracy (WHNND). It is known that the Milnor number $\mu$ resp. the delta-invariant $\delta$ can be computed by explicit formulas $\mu_{N}$ resp. $\delta_{N}$ from the Newton diagram of $f$ if $f$ is NND resp. WNND. It was however unknown whether the equalities $\mu=\mu_{N}$ resp. $\delta=\delta_{N}$ can be characterized by a certain non-degeneracy condition on $f$ and, if so, by which one. We show that $\mu=\mu_{N}$ resp. $\delta=\delta_{N}$ is equivalent to INND resp. WHNND and give some applications and interesting examples related to the existence of "wild vanishing cycles". Although the results are new in any characteristic, the main difficulties arise in positive characteristic.
\end{abstract}

\section{Introduction}

Let $K$ be an algebraically closed field, $K[[x]]=K\left[\left[x_{1}, \ldots, x_{n}\right]\right]$ the formal power series ring and $\mathfrak{m}$ its maximal ideal. Let us recall the definition of the Newton diagram and Wall's notion of a $C$-polytope (see [Wal99]). To each power series $f=\sum_{\alpha} c_{\alpha} x^{\alpha} \in K[[x]]$ we can associate its Newton polyhedron $\Gamma_{+}(f)$ as the convex hull of the set

$$
\bigcup_{\alpha \in \operatorname{supp}(f)}\left(\alpha+\mathbb{R}_{\geq 0}^{n}\right)
$$

where $\operatorname{supp}(f)=\left\{\alpha \mid c_{\alpha} \neq 0\right\}$ denotes the support of $f$. This is an unbounded polytope in $\mathbb{R}^{n}$. We call the union $\Gamma(f)$ of its compact faces the Newton diagram of $f$. By $\Gamma_{-}(f)$ we denote the union of all line segments joining the origin to a point on $\Gamma(f)$. We always assume that $f \in \mathfrak{m}$ if not explicitly stated otherwise.

If the Newton diagram of a singularity $f$ meets all coordinate axes we call $f$ convenient. However, not every isolated singularity is convenient, and one then has to enlarge the Newton diagram. A compact rational polytope $P$ of dimension $n-1$ in the positive orthant $\mathbb{R}_{\geq 0}^{n}$ is called a $C$-polytope if the region above $P$ is convex and if every ray in the positive orthant emanating from the origin meets $P$ in exactly one point. The Newton diagram of $f$ is a $C$-polytope iff $f$ is convenient.

Date: June 4, 2018.

1991 Mathematics Subject Classification. Primary 14B05, 32S10, 32S25, 58K40..

Key words and phrases. Milnor number, delta-invariant, Newton non-degenerate, inner Newton non-degenerate, weak Newton non-degenerate. 
We first introduce the different notions of non-degeneracy. For this let $f=$ $\sum_{\alpha} c_{\alpha} x^{\alpha} \in \mathfrak{m}$ be a power series, let $P$ be a $C$-polytope and let $\Delta$ be a face of $P$. By $f_{\Delta}:=\operatorname{in}_{\Delta}(f):=\sum_{\alpha \in \Delta} c_{\alpha} x^{\alpha}$ we denote the initial form or principal part of $\mathrm{f}$ along $\Delta$. Following Kouchnirenko we call $f$ non-degenerate ND along $\Delta$ if the Jacobian idea $1 \mathrm{j}\left(f_{\Delta}\right)$ has no zero in the torus $\left(K^{*}\right)^{n} . f$ is then said to be Newton non-degenerate NND if $f$ is non-degenerate along each face (of any dimension) of the Newton diagram $\Gamma(f)$. We do not require $f$ to be convenient.

To define inner non-degeneracy we need to fix two more notions. The face $\Delta$ is an inner face of $P$ if it is not contained in any coordinate hyperplane. Each point $q \in K^{n}$ determines a coordinate hyperspace $H_{q}=\bigcap_{q_{i}=0}\left\{x_{i}=0\right\} \subset \mathbb{R}^{n}$ in $\mathbb{R}^{n}$. We call $f$ inner non-degenerate IND along $\Delta$ if for each zero $q$ of the Jacobian ideal $\mathrm{j}\left(\operatorname{in}_{\Delta}(f)\right)$ the polytope $\Delta$ contains no point on $H_{q} . \quad f$ is called inner Newton non-degenerate INND w.r.t. a $C$-polytope $P$ if no point of $\operatorname{supp}(f)$ lies below $P$ and $f$ is IND along each inner face of $P$. We call $f$ simply inner Newton non-degenerate INND if it is INND w.r.t some $C$-polytope.

Finally, we call $f$ weakly non-degenerate WND along $\Delta$ if the Tjurina idea 2 $t j\left(\operatorname{in}_{\Delta}(f)\right)$ has no zero in the torus $\left(K^{*}\right)^{n}$, and $f$ is called weakly Newton nondegenerate WNND if $\mathrm{f}$ is weakly non-degenerate along each top-dimensional face of $\Gamma(f)$. Note that NND implies WNND while NND does not imply INND and vice versa. See [BGM10, Remark 3.1] for facts on and relations between the different types of non-degeneracy.

For any compact polytope $Q$ in $\mathbb{R}_{\geq 0}^{n}$ we denote by $V_{k}(Q)$ the sum of the $k$ dimensional Euclidean volumes of the intersections of $Q$ with the $k$-dimensional coordinate subspaces of $\mathbb{R}^{n}$ and, following Kouchnirenko, we then call

$$
\mu_{N}(Q)=\sum_{k=0}^{n}(-1)^{n-k} k ! V_{k}(Q)
$$

the Newton number of $Q$. For a power series $f \in K[[x]]$ we define the Newton number of $f$ to be

$$
\mu_{N}(f)=\sup \left\{\mu_{N}\left(\Gamma_{-}\left(f_{m}\right)\right) \mid f_{m}:=f+x_{1}^{m}+\ldots+x_{n}^{m}, m \geq 1\right\} .
$$

If $f$ is convenient then

$$
\mu_{N}(f)=\mu_{N}\left(\Gamma_{-}(f)\right) .
$$

The following theorem was proved by Kouchnirenko in arbitrary characteristic. We recall that $\mu(f):=\operatorname{dim} K[[x, y]] / j(f)$ is the Milnor number of $f$.

Theorem 1.1. [Kou76] For $f \in K[[x]]$ we have $\mu_{N}(f) \leq \mu(f)$, and if $f$ is $N N D$ and convenient then $\mu_{N}(f)=\mu(f)<\infty$.

\footnotetext{
${ }^{1}$ The Jacobian ideal $j(f)$ denotes the ideal generated by all partials of $f \in K[[x]]$.

${ }^{2}$ For $f \in K[[x]]$ we call $t j(f)=\langle f\rangle+j(f)$ the Tjurina ideal of $f$.
} 
Since Theorem 1.1 does not cover all semi-quasihomogeneous singularities, Wall introduced the condition INND (denoted by NPND* in Wal99]). Using Theorem 1.1. Wall proved the following theorem for $K=\mathbb{C}$ which was extended to arbitrary $K$ in BGM10.

Theorem 1.2. Wal99, [BGM10] If $f \in K[[x]]$ is INND, then

$$
\mu(f)=\mu_{N}(f)=\mu_{N}\left(\Gamma_{-}(f)\right)<\infty .
$$

Kouchnirenko proved that the condition "convenient" is not necessary in Theorem 1.1 if $\operatorname{char}(K)=0$. The authors in [BGM10] show that in the planar case Kouchnirenko's result holds in arbitrary characteristic without the assumption that $f$ is convenient (allowing $\mu(f)=\infty$ ):

Proposition 1.3. [BGM10, Proposition 4.5] Suppose that $f \in K[[x, y]]$ is $N N D$, then $\mu_{N}(f)=\mu(f)$.

\section{Milnor NUmBer}

In the following we consider only the case of plane curve singularities. The main result of this section says that for $f \in K[[x, y]]$, the condition $\mu(f)=\mu_{N}(f)<\infty$ is equivalent to $f$ being INND (Theorem 2.13). In characteristic zero this is also equivalent to $f$ being NND and $\mu_{N}(f)<\infty$ (Corollary 2.17). However, in positive characteristic, this is in general not true as the following example shows.

Example 2.1. $f=x^{3}+x y+y^{3}$ in characteristic 3 satisfies $\mu(f)=\mu_{N}(f)=1$ but $f$ is not NND.

Remark 2.2. Let $f \in K[[x, y]]$ be convenient and $A_{i}=\left(c_{i}, e_{i}\right), i=0, \ldots, k$ the vertices of $\Gamma(f)$ with $c_{0}=e_{k}=0, c_{i}<c_{i+1}$ and $e_{i}>e_{i+1}$. Then

$$
\mu_{N}(f)=2 V_{2}\left(\Gamma_{-}(f)\right)-c_{k}-e_{0}+1
$$

Lemma 2.3. Let $f, g \in K[[x, y]]$ be convenient such that $\Gamma_{-}(f) \subset \Gamma_{-}(g)$. Then

(a) $\mu_{N}(f) \leq \mu_{N}(g)$.

(b) The equality holds if and only if $\Gamma_{-}(f) \cap \mathbb{R}_{\geq 1}^{2}=\Gamma_{-}(g) \cap \mathbb{R}_{\geq 1}^{2}$, where

$$
\mathbb{R}_{\geq 1}^{2}=\left\{(x, y) \in \mathbb{R}^{2} \mid x \geq 1, y \geq 1\right\}
$$

Part (a) of the lemma was also shown in Biv09, Coro. 5.6]. Let us denote by $\Gamma_{1}(f)$ the cone joining the origin with $\Gamma(f) \cap \mathbb{R}_{\geq 1}^{2}$. (cf. Fig. 1). 


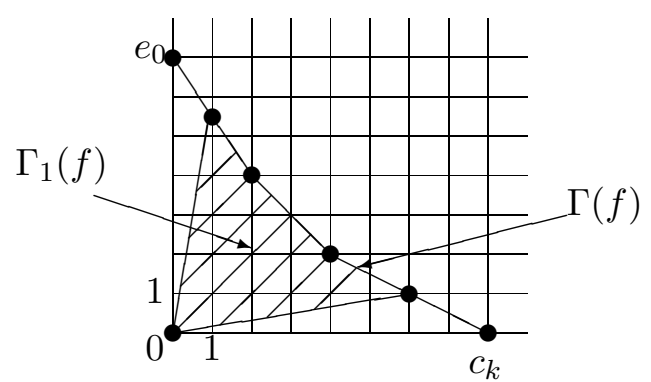

Fig. 1.

Proof. First, we prove that

$$
\mu_{N}(f)=V_{2}\left(\Gamma_{1}(f)\right)+1 .
$$

It is easy to see that $\Gamma_{1}(f)$ divides $\Gamma_{-}(f)$ into three parts whose volumes are $c_{k} / 2, V_{2}\left(\Gamma_{1}(f)\right)$ and $e_{0} / 2$. Therefore

$$
\mu_{N}(f)=2 V_{2}\left(\Gamma_{-}(f)\right)-c_{k}-e_{0}+1=2 V_{2}\left(\Gamma_{1}(f)\right)+1 .
$$

(a) Clearly, if $\Gamma_{-}(f) \subset \Gamma_{-}(g)$ then $\Gamma_{1}(f) \subset \Gamma_{1}(g)$ and hence

$$
\mu_{N}(f)=V_{2}\left(\Gamma_{1}(f)\right)+1 \leq 2 V_{2}\left(\Gamma_{1}(g)\right)+1=\mu_{N}(g) .
$$

(b) follows easily from the formula $\mu_{N}(f)=2 V_{2}\left(\Gamma_{1}(f)\right)+1$.

We recall some classical notions. Let $f \in K[[x, y]]$ be irreducible. A couple $(x(t), y(t)) \in K[[t]]^{2}$ is called a (primitive) parametrization of $f$, if $f(x(t), y(t))=$ 0 and if the following universal factorization property holds: for each $(u(t), v(t)) \in$ $K[[t]]^{2}$ with $f(u(t), v(t))=0$, there exists a unique series $h(t) \in K[[t]]$ such that $u(t)=x(h(t))$ and $v(t)=y(h(t))$.

If $g \in K[[x, y]]$ is irreducible and $(x(t), y(t))$ its parametrization, then the intersection multiplicity of any $f \in K[[x, y]]$ with $g$ is given by $i(f, g)=\operatorname{ord} f(x(t), y(t))$, and if $u$ is a unit then $i(f, u)=0$. The intersection multiplicity of $f$ with a reducible power series $g=g_{1} \cdot \ldots \cdot g_{s}$ is defined to be the sum $i(f, g)=$ $i\left(f, g_{1}\right)+\ldots+i\left(f, g_{s}\right)$.

Proposition 2.4. [GLS06, Pro. 3.12] Let $f, g \in K[[x, y]]$. Then

$$
i(f, g)=i(g, f)=\operatorname{dim} K[[x, y]] /\langle f, g\rangle .
$$

The proof in GLS06 was given for $K=\mathbb{C}$ but works in any characteristic.

Let $f=\sum_{i, j} c_{i j} x^{i} y^{j} \in K[[x, y]]$ and $\Gamma(f)$ be its Newton diagram. We call

$$
f_{\text {in }}:=\sum_{(i, j) \in \Gamma(f)} c_{i j} x^{i} y^{j}
$$

the initial part of $f$. 
Proposition 2.5. Let $f \in \mathfrak{m} \subset K[[x, y]]$ be irreducible, $x$-general of order $m$ and $y$-general of order $n$. Let $(x(t), y(t))$ be parametrization of $f$. Then

(a) $\operatorname{ord}(x(t))=n$ and $\operatorname{ord}(y(t))=m$.

(b) The Newton diagram of $f$ is the straight line segment.

(c) There exist $\xi, \lambda \in K^{*}$ such that

$$
f_{\text {in }}(x, y)=\xi \cdot\left(x^{m / q}-\lambda y^{n / q}\right)^{q},
$$

where $q=(m, n)$.

Proof. cf. Cam80, Lemma 3.4.3, 3.4.4, 3.4.5].

Proposition 2.6. BrK86, Lemma 3] Let $f \in K[[x, y]]$ and let $E_{i}, i=1, \ldots, k$ be the edges of its Newton diagram. Then there is a factorization of $f$ :

$$
f=\operatorname{monomial} \cdot \bar{f}_{1} \cdot \ldots \cdot \bar{f}_{k}
$$

such that $\bar{f}_{i}$ is convenient, $f_{E_{i}}=$ monomial $\times\left(\bar{f}_{i}\right)_{\text {in }}$. In particular, if $f$ is convenient then $f=\bar{f}_{1} \cdot \ldots \cdot \bar{f}_{k}$.

A polynomial $f=\sum_{i, j} c_{i j} x^{i} y^{j} \in K[x, y]$ is called weighted homogeneous or quasihomogeneous of type $(n, m ; d)$ if $m, n, d$ are positive integers satisfying $n i+$ $m j=d$, for each $(i, j) \in \operatorname{supp}(f)$.

Let $f \in K[[x, y]]$ be a formal power series and $n, m$ positive integers. We can decompose $f$ into a sum

$$
f=f_{d}^{\mathrm{w}}+f_{d+1}^{\mathrm{w}}+\ldots,
$$

where $f_{d}^{\mathrm{w}} \neq 0$ and $f_{l}^{\mathrm{w}}$ is weighted homogeneous of type $(n, m ; l)$ for $l \geq d$. We call $f_{d}^{\mathrm{w}}$ the first term of the decomposition.

For each series $\varphi(t)=c_{1} t^{\alpha_{1}}+c_{2} t^{\alpha_{2}}+\ldots$ with $c_{1} \neq 0, \alpha_{1}<\alpha_{2}<\ldots$, we set

$$
L T(\varphi(t)):=c_{1} t^{\alpha_{1}} \text { and } L C(\varphi(t)):=c_{1} .
$$

Lemma 2.7. Let $m, n$ be two positive integers. Let $x(t), y(t) \in K[[t]]$ with $\operatorname{LT}(x(t))=a t^{\alpha}$ and $\operatorname{LT}(y(t))=b t^{\beta}$ such that $\alpha: \beta=n: m$. Let $f=$ $f_{d}^{\mathrm{w}}+f_{d+1}^{\mathrm{w}}+\ldots$, be a $(n, m)$-weighted homogeneous decomposition of $f \in K[[x, y]]$. Then ord $f(x(t), y(t)) \geq \frac{d \alpha}{n}$. Equality holds if and only if $f_{d}(a, b) \neq 0$.

Proof. We can write $x(t)=t^{\alpha}(a+u(t))$ and $y(t)=t^{\beta}(b+v(t))$, where ord $u(t)>0$ and $\operatorname{ord} v(t)>0$. Then

$$
\begin{aligned}
f_{l}^{\mathrm{w}}(x(t), y(t)) & =\sum_{n i+m j=l} c_{i j}\left(t^{\alpha}(a+u(t))\right)^{i}\left(t^{\beta}(b+v(t))\right)^{j} \\
& =t^{\frac{l \alpha}{n}} f_{l}^{\mathrm{w}}(a+u(t), b+v(t)) .
\end{aligned}
$$

Thus ord $f_{l}^{\mathrm{w}}(x(t), y(t)) \geq \frac{l \alpha}{n}$ and hence ord $f(x(t), y(t)) \geq \frac{d \alpha}{n}$. 
Since $f_{d}^{\mathrm{w}}(a+u(t), b+v(t))=f_{d}^{\mathrm{w}}(a, b)+t h(t)$ for some power series $h$,

$$
\operatorname{ord} f(x(t), y(t))=\operatorname{ord} f_{d}^{\mathrm{w}}(x(t), y(t))=\frac{d \alpha}{n}
$$

iff $f_{d}^{\mathrm{w}}(a, b) \neq 0$.

Lemma 2.8. Let $f \in K[[x, y]]$ be convenient such that $\Gamma(f)$ has only one edge. Let $m=\operatorname{ord} f(x, 0), n=\operatorname{ord} f(0, y)$ and $f=f_{1} \cdot \ldots \cdot f_{r}$ a factorization of $f$ into its branches (irreducible factors).

(a) Let $\left(x_{j}(t), y_{j}(t)\right)$ be a parametrization of $f_{j}, j=1, \ldots, r$ with $\operatorname{LT}\left(x_{j}(t)\right)=$ $a_{j} t^{\alpha_{j}}$ and $\operatorname{LT}\left(y_{j}(t)\right)=b_{j} t^{\beta_{j}}$. Then $f_{\text {in }}\left(a_{j}, b_{j}\right)=0, \alpha_{j}: \beta_{j}=n: m$ and $\alpha_{1}+\cdots+\alpha_{r}=n$.

(b) Let $a, b \in K^{*}$ such that $f_{\text {in }}(a, b)=0$. Then there is a parametrization $(x(t), y(t))$ of a branch of $f$ satisfying $\mathrm{LC}(x(t))=a$ and $\operatorname{LC}(y(t))=b$.

Proof. Let $f=f_{d}^{\mathrm{w}}+f_{d+1}^{\mathrm{w}}+\ldots$ with $f_{d}^{\mathrm{w}} \neq 0$ be the $(n, m)$-weighted homogeneous decomposition of $f$. Then $f_{d}^{\mathrm{w}}=f_{\text {in }}$.

(a) It is easily verified that $f_{\text {in }}=\prod\left(f_{j}\right)_{\text {in }}$ then $\left(f_{j}\right)_{\text {in }}$ is also a $(n, m)$-weighted homogeneous polynomial of order some $d_{j}$. By Proposition 2.5, ord $f_{j}(x, 0)=\beta_{j}$ and $\operatorname{ord} f_{j}(0, y)=\alpha_{j}$, i.e. $x^{\beta_{j}}$ and $y^{\alpha_{j}}$ are monomials of $\left(f_{j}\right)_{\text {in. Thus }} n \beta_{j}=d_{j}=$ $m \alpha_{j}$ and hence $\alpha_{j}: \beta_{j}=n: m$.

Since $f\left(x_{j}(t), y_{j}(t)\right)=0$, i.e. ord $f\left(x_{j}(t), y_{j}(t)\right)=+\infty>\frac{d \alpha_{j}}{n}$, Lemma 2.7yields that $f_{d}^{\mathrm{w}}\left(a_{j}, b_{j}\right)=0$, i.e. $f_{\text {in }}\left(a_{j}, b_{j}\right)=0$.

Now, by the definition of intersection multiplicity we have

$$
n=i(f, x)=\sum_{j=1}^{r} \operatorname{ord} x_{j}(t)=\sum_{j=1}^{r} \alpha_{j} .
$$

(b) Since $f=g \cdot h$ implies $f_{\text {in }}=g_{\text {in }} \cdot h_{\text {in }}$, it suffices to prove part (b) for the irreducible case. Then by Proposition 2.5(c), there exist $\xi, \lambda \in K^{*}$ such that

$$
f_{i n}(x, y)=\rho \cdot\left(x^{m^{\prime}}-\lambda y^{n^{\prime}}\right)^{q},
$$

where $q=(m, n) ; m^{\prime}=m / q$ and $n^{\prime}=n / q$. Clearly, $\left(m^{\prime}, n^{\prime}\right)=1$. Then it is impossible for the characteristic $p$ of $K$ divides both $m^{\prime}$ and $n^{\prime}$. We may assume that $p$ does not divide $n^{\prime}$.

Let $(\bar{x}(t), \bar{y}(t))$ be a parametrization of $f$. It follows from Proposition 2.5 that $\operatorname{LT}(\bar{x}(t))=\bar{a} t^{n}$ and $\operatorname{LT}(\bar{y}(t))=\bar{b} t^{m}$ for some $\bar{a}, \bar{b} \in K^{*}$ satisfying $f_{\text {in }}(\bar{a}, \bar{b})=0$.

Set

$$
g(y):=a^{m^{\prime}}-\lambda y^{n^{\prime}} \text { and } \sqrt[n^{\prime}]{a / \bar{a}}:=\left\{\xi_{i} \mid i=1, \ldots, n^{\prime}\right\}
$$

Then

$$
g\left(\bar{b} \xi_{i}^{m^{\prime}}\right)=f_{\text {in }}\left(a, \bar{b} \xi_{i}^{m^{\prime}}\right)=f_{\text {in }}\left(\bar{a} \xi_{i}^{n}, \bar{b} \xi_{i}^{m^{\prime}}\right)=\xi_{i}^{d} f_{\text {in }}(\bar{a}, \bar{b})=0
$$


Since $\left(m^{\prime}, n^{\prime}\right)=1$, it is easy to see that $\xi_{i}^{m^{\prime}} \neq \xi_{j}^{m^{\prime}}$ for all $i \neq j$. Thus the set

$$
\left\{\bar{b} \xi_{i}^{m^{\prime}} \mid i=1, \ldots, n^{\prime}\right\}
$$

contains all of roots of $g$. Since $0=f_{i n}(a, b)=\rho \cdot g(b)^{q}, g(b)=0$. Then there is an index $i_{0}$ such that $b=\bar{b} \xi_{i_{0}}^{m^{\prime}}$. Choose a $\epsilon$ in $\sqrt[q]{\xi_{i_{0}}}$ and put

$$
x(t)=\bar{x}(\epsilon t) \text { and } y(t)=\bar{y}(\epsilon t),
$$

we get $\mathrm{LC}(x(t))=a$ and $\mathrm{LC}(y(t))=b$.

Definition 2.9. Let $f=\sum c_{i j} x^{i} y^{j} \in K[[x, y]]$ be such that $(0, n)$ is the vertex on the $y$-axis of $\Gamma(f)$. Let $\left(1, j_{1}\right)$ be the intersection point of $\Gamma(f)$ and the line $x=1$. We define $f$ to be $N D 1$ along $(0, n)$ if either $\operatorname{char}(K)=p=0$ or if $p \neq 0$ then $p \not \mid n$ or $j_{1} \in \mathbb{N}$ and the coefficient $c_{1 j_{1}}$ of $x y^{j_{1}}$ in $f$ is different from zero. ND1 along $(m, 0)$, with $(m, 0)$ the vertex on the $x$-axis of $\Gamma(f)$, is defined analogously.

$f$ is called NND1 if $f$ is convenient, ND along each inner face and ND1 along each vertex on the axes of $\Gamma(f)$.

Proposition 2.10. Let $f=\sum c_{i j} x^{i} y^{j} \in K[[x, y]]$ be convenient and let $(0, n)$ (resp. $(m, 0))$ be the vertex on the $y$-axis (resp. on the $x$-axis) of $\Gamma(f)$. Assume that $f$ is not ND1 along the point $(0, n)$ or $(m, 0)$ then $\mu(f)>\mu_{N}(f)$.

Proof. We consider only $(0, n)$ since $(m, 0)$ is analogous. Let $\left(1, j_{1}\right)$ be the intersection point of $\Gamma(f)$ and the line $x=1$. The assumption that $f$ is not ND1 along the point $(0, n)$ implies that $p \mid n$ and $c_{1 j_{1}}=0$. Putting $g(x, y)=f(x, y)-c_{0 n} y^{n}$ one then has $\mu(f)=\mu(g)$ and $\Gamma_{-}(f) \subset \Gamma_{-}(g)$. On the other hand, it is easy to see that $\left(1, j_{1}\right) \in \Gamma_{+}(f) \backslash \Gamma_{+}(g)$. This means $\Gamma_{-}(f) \cap \mathbb{R}_{\geq 1}^{2} \varsubsetneqq \Gamma_{-}(g) \cap \mathbb{R}_{\geq 1}^{2}$. It hence follows from Lemma 2.3 that $\mu_{N}(g)>\mu_{N}(f)$. Thus

$$
\mu(f)=\mu(g) \geq \mu_{N}(g)>\mu_{N}(f) .
$$

Proposition 2.11. Let $f \in K[[x, y]]$ be convenient. If $f$ is degenerate along some inner vertex of $\Gamma(f)$ then $\mu(f)>\mu_{N}(f)$.

Proof. Assume that $f$ is degenerate along some vertex $\left(i_{0}, j_{0}\right)$ of $\Gamma(f)$ with $i_{0}>0$ and $j_{0}>0$. Then $p \neq 0$ and $i_{0}$ and $j_{0}$ are divisible by $p$. Put $g(x, y)=$ $f(x, y)-c_{i_{0} j_{0}} x^{i_{0}} y^{j_{0}}$, then $j(f)=j(g)$ and hence $\mu(f)=\mu(g)$. Clearly, $\Gamma_{+}(g)$ does not contain the point $\left(i_{0}, j_{0}\right)$. Thus

$$
\Gamma_{-}(f) \cap \mathbb{R}_{\geq 1}^{2} \varsubsetneqq \Gamma_{-}(g) \cap \mathbb{R}_{\geq 1}^{2} .
$$

Lemma 2.3 hence implies that $\mu_{N}(g)>\mu_{N}(f)$. We then have

$$
\mu(f)=\mu(g) \geq \mu_{N}(g)>\mu_{N}(f) .
$$


Proposition 2.12. Let $f \in K[[x, y]]$ be convenient. If $f$ is degenerate along some edge of $\Gamma(f)$ then $\mu(f)>\mu_{N}(f)$.

Proof. Let $f(x, y)=\sum c_{\alpha \beta} x^{\alpha} y^{\beta}$. Let $f_{x}, f_{y}$ be the partials of $f$ and put $h(x, y):=$ $x f_{x}(x, y)+\lambda y f_{y}(x, y)$, where $\lambda \in K$ is generic. Then

$$
h(x, y)=\sum(\alpha+\lambda \beta) c_{\alpha \beta} x^{\alpha} y^{\beta} .
$$

Thus $\operatorname{supp}(h)=\operatorname{supp}(f) \backslash(p \mathbb{N})^{2}$ and if $p=0$ then $\operatorname{supp}(h)=\operatorname{supp}(f)$. Hence $\Gamma_{+}(h) \subset \Gamma_{+}(f)$.

Case 1: $f$ is ND along each vertex of $\Gamma(f)$.

Assume now that $(i, j)$ is a vertex of $\Gamma(f)$. Since $f$ is ND along $(i, j), p=0$ or $p \neq 0$ and one of $i, j$ is not divisible by $p$. Therefore $(i, j) \in \operatorname{supp}(f) \backslash(p \mathbb{N})^{2}=$ $\operatorname{supp}(h)$ and then $\Gamma_{+}(f) \subset \Gamma_{+}(h)$. Hence $\Gamma(h)=\Gamma(f)$.

Let $E_{i}, i=1, \ldots, k$ be edges of $\Gamma(h)$. By Proposition 2.6, we can write $h=$ $\bar{h}_{1} \ldots \bar{h}_{k}$, where $\bar{h}_{i}$ are convenient and $\left.h_{E_{i}}(x, y)\right)=$ monomial $\times\left(\bar{h}_{i}\right)_{i n}$. We denote by $m_{i}$ and $n_{i}$ the lengths of the projections of $E_{i}$ on the horizontal and vertical axes.

Let $h=h_{d_{i}}^{\mathrm{w}}+h_{d_{i}+1}^{\mathrm{w}}+\ldots$ with $h_{d_{i}}^{\mathrm{w}} \neq 0$ be the $\left(n_{i}, m_{i}\right)$-weighted homogeneous decomposition of $h$. Then $h_{d_{i}}^{\mathrm{w}}=h_{E_{i}}$. Since $E_{i}$ is also an edge of $\Gamma(f), f=$ $f_{d_{i}}^{\mathrm{w}}+f_{d_{i}+1}^{\mathrm{w}}+\ldots$ is the $\left(n_{i}, m_{i}\right)$-weighted homogeneous decomposition of $f$ with $f_{d_{i}}^{\mathrm{w}}=f_{E_{i}}$ and then

$$
h_{d_{i}}^{\mathrm{w}}=\sum_{n_{i} \alpha+m_{i} \beta=d_{i}}(\alpha+\lambda \beta) c_{\alpha \beta} x^{\alpha} y^{\beta}=x \frac{\partial f_{d_{i}}^{\mathrm{w}}}{\partial x}+\lambda y \frac{\partial f_{d_{i}}^{\mathrm{w}}}{\partial y} .
$$

Let $y f_{y}=g_{d_{i}{ }^{\prime}}^{\mathrm{w}}+g_{d_{i}{ }^{\prime}+1}^{\mathrm{w}}+\ldots$ be the $\left(n_{i}, m_{i}\right)$-weighted homogeneous decomposition of $y f_{y}$. It is easy to see that $d_{i}{ }^{\prime} \geq d_{i}$ and $d_{i}{ }^{\prime}=d_{i}$ iff $y \frac{\partial f_{d_{i}}^{\mathrm{w}}}{\partial y} \neq 0$.

Claim 1. Let $A_{i-1}, A_{i}$ be the vertices of the edge $E_{i}$ and let $V_{2}\left(O A_{i-1} A_{i}\right)$ be the volume of triangle $O A_{i-1} A_{i}$. Then $d_{i}=2 V_{2}\left(O A_{i-1} A_{i}\right)$.

Proof. Let $\left(c_{i}, e_{i}\right)$ be the coordinates of $A_{i}, i=0, \ldots, k$. Then $m_{i}=c_{i}-c_{i-1}$ and $n_{i}=e_{i-1}-e_{i}$. (cf. Fig. 2).

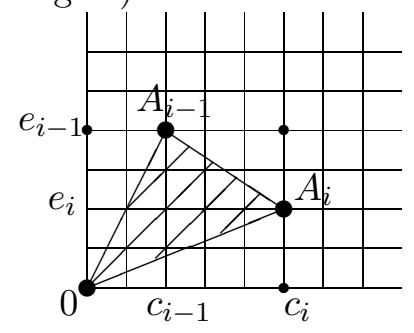

Fig. 2. 
Considering the rectangle $(0,0) ;\left(c_{i}, 0\right) ;\left(c_{i}, e_{i-1}\right) ;\left(0, e_{i-1}\right)$ we have

$$
\begin{aligned}
2 V_{2}\left(O A_{i-1} A_{i}\right) & =2 c_{i} e_{i-1}-c_{i} e_{i}-c_{i-1} e_{i-1}-m_{i} n_{i} \\
& =\left(c_{i-1}+m_{i}\right) e_{i-1}+c_{i}\left(e_{i}+n_{i}\right)-c_{i} e_{i}-c_{i-1} e_{i-1}-m_{i} n_{i} \\
& =m_{i} e_{i-1}+c_{i} n_{i}-m_{i} n_{i}=m_{i}\left(e_{i}+n_{i}\right)+c_{i} n_{i}-m_{i} n_{i} \\
& =m_{i} e_{i}+n_{i} c_{i}=d_{i}
\end{aligned}
$$

This proves Claim 1.

Claim 2. $i\left(\bar{h}_{i}, y f_{y}\right) \geq d_{i}$, and if $f$ is degenerate along $E_{i}$ then $i\left(\bar{h}_{i}, y f_{y}\right)>d_{i}$.

Proof. Let $\left(x_{j}(t), y_{j}(t)\right), j=1, \ldots, r$ be parametrizations of the branches $\bar{h}_{i, j}$ of $\bar{h}_{i}$. Then by Lemma 2.8, we have $\operatorname{LT}\left(x_{j}(t)\right)=a_{j} t^{\alpha_{j}}$ and $\operatorname{LT}\left(y_{j}(t)\right)=b_{j} t^{\beta_{j}}$, where $a_{j}, b_{j} \in K^{*}, \bar{h}_{i}\left(a_{j}, b_{j}\right)=0, \alpha_{j}: \beta_{j}=n_{i}: m_{i}$ for all $j=1, \ldots, r$ and $\alpha_{1}+\ldots+\alpha_{r}=n_{i}$. It follows from Lemma 2.7 that ord $\left(y f_{y}\right)\left(x_{j}(t), y_{j}(t)\right) \geq \frac{d_{i}{ }^{\prime} \alpha_{j}}{n_{i}}$ for all $j=1, \ldots, r$. Thus

$$
i\left(\bar{h}_{i}, y f_{y}\right)=\sum_{j=1}^{r} \operatorname{ord}\left(y f_{y}\right)\left(x_{j}(t), y_{j}(t)\right) \geq \sum_{j=1}^{r} \frac{d_{i}{ }^{\prime} \alpha_{j}}{n_{i}}=d_{i}{ }^{\prime} \geq d_{i} .
$$

Assume that $f$ is degenerate along $E_{i}$ then there exist $a, b \neq 0$ such that

$$
x \frac{\partial f_{d_{i}}^{\mathrm{w}}}{\partial x}(a, b)=y \frac{\partial f_{d_{i}}^{\mathrm{w}}}{\partial y}(a, b)=0 .
$$

Therefore $h_{d_{i}}(a, b)=0$. Lemma 2.8 implies that there is a parametrization of a branch of $\bar{h}_{i}$ such that $\operatorname{LT}(\bar{x}(t))=a t^{\alpha}$ and $\operatorname{LT}(\bar{y}(t))=b t^{\beta}$. We may assume that $(\bar{x}(t), \bar{y}(t))$ is a parametrization of the branch $\bar{h}_{i, 1}$. Then $\alpha=\alpha_{1}$ and $\beta=\beta_{1}$.

To show $i\left(\bar{h}_{i}, y f_{y}\right)>d_{i}$, we may restrict to the case that $d_{i}{ }^{\prime}=d_{i}$, because of the inequality $i\left(\bar{h}_{i}, y f_{y}\right) \geq d_{i}{ }^{\prime} \geq d_{i}$. As $d_{i}{ }^{\prime}=d_{i}$ then $g_{d_{i}{ }^{\prime}}^{\mathrm{w}}(a, b)=y \frac{\partial f_{d_{i}}^{\mathrm{w}}}{\partial y}(a, b)=0$. Lemma 2.7 yields

$$
\operatorname{ord}\left(y f_{y}\right)(\bar{x}(t), \bar{y}(t))>\frac{d_{i} \alpha_{1}}{n_{i}}
$$

Thus

$$
\begin{aligned}
i\left(\bar{h}_{i}, y f_{y}\right) & =\operatorname{ord}\left(y f_{y}\right)(\bar{x}(t), \bar{y}(t))+\sum_{j=2}^{r} \operatorname{ord}\left(y f_{y}\right)\left(x_{j}(t), y_{j}(t)\right) \\
& >\frac{d_{i} \alpha_{1}}{n_{i}}+\sum_{j=2}^{r} \frac{d_{i}{ }^{\prime} \alpha_{j}}{n_{i}}=d_{i}
\end{aligned}
$$

This proves Claim 2.

It now follows from Claim 1 and Claim 2 that

$$
i\left(h, y f_{y}\right) \geq \sum_{i=1}^{k} 2 V_{2}\left(O A_{i-1} A_{i}\right)=2 V_{2}\left(\Gamma_{-}(f)\right) .
$$


Hence

$$
\begin{aligned}
\mu(f)=i\left(f_{x}, f_{y}\right) & =i\left(h, y f_{y}\right)-i\left(x, f_{y}\right)-i\left(f_{x}, y\right)-1 \\
& \geq 2 V_{2}\left(\Gamma_{-}(f)\right)-\left(e_{0}-1\right)-\left(c_{k}-1\right)-1 \\
& =\mu_{N}(f) .
\end{aligned}
$$

Moreover, if $f$ is degenerate along some edge of $\Gamma(f)$ then $\mu(f)>\mu_{N}(f)$ by Claim 1 and 2. This proves of Case 1.

Case 2: In the general case, by propositions [2.10, 2.11 we may assume that $f$ is ND along each inner vertex and ND1 along the two vertice on the axes of $\Gamma(f)$. For $m$ sufficiently large and $p \nmid{ }_{m}$, we put

$$
\bar{f}_{m}(x, y)=\sum_{(\alpha, \beta) \notin(p \mathbb{N})^{2}} c_{\alpha \beta} x^{\alpha} y^{\beta}+x^{m}+y^{m} .
$$

Then

$$
\mu\left(\bar{f}_{m}\right)=\mu\left(f_{m}\right)=\mu(f) \text { and } \mu_{N}\left(\bar{f}_{m}\right) \geq \mu_{N}\left(f_{m}\right)=\mu_{N}(f),
$$

where the inequality follows from Lemma 2.3 .

Claim 3. $\bar{f}_{m}$ is degenerate along some edge of $\Gamma(\bar{f})$.

Proof. By the assumption $f$ is degenerate along some edge $E$ of $\Gamma(f)$. If $E$ is also an edge of $\Gamma\left(\bar{f}_{m}\right)$ then $j\left(\operatorname{in}_{E}\left(\bar{f}_{m}\right)=j\left(\operatorname{in}_{E}(f)\right)\right.$ and hence $\bar{f}_{m}$ is degenerate along $E$. If $E$ is not an edge of $\Gamma\left(\bar{f}_{m}\right)$, then $E$ must meet the axes since $f$ is ND along each inner vertex of $\Gamma(f)$. We may assume that $(0, n)$ is a vertex of $E$. We will show that

$$
\sharp\left(\operatorname{supp}\left(\bar{f}_{m}\right) \cap E\right) \geq 2 .
$$

Let $\left(1, j_{1}\right)$ be the intersection point of $E$ and the line $x=1$. Since $f$ is ND1 along $(0, n)$, either $(0, n) \in \operatorname{supp}\left(\bar{f}_{m}\right) \cap E$ or $\left(1, j_{1}\right) \in \operatorname{supp}\left(\bar{f}_{m}\right) \cap E$, i.e. $\operatorname{supp}\left(\bar{f}_{m}\right) \cap E \neq \emptyset$ On the other hand, it is easy to see that $\sharp\left(\operatorname{supp}\left(\bar{f}_{m}\right) \cap E\right) \neq 1$ since $f$ is degenerate along the edge $E$. Hence $\sharp\left(\operatorname{supp}\left(\bar{f}_{m}\right) \cap E\right) \geq 2$. Let us denote by $\bar{E}$ the convex hull of the set $\operatorname{supp}\left(\bar{f}_{m}\right) \cap E$. Then $\bar{E}$ is an edge of $\Gamma\left(\bar{f}_{m}\right)$ and $j\left(\operatorname{in}_{\bar{E}}\left(\bar{f}_{m}\right)=j\left(\operatorname{in}_{E}(f)\right)\right.$. Thus $\bar{f}_{m}$ is degenerate along $\bar{E}$ since $f$ is degenerate along the edge $E$, which proves Claim 3.

Now, by definition, $\bar{f}_{m}$ is ND along each vertex of $\Gamma\left(\bar{f}_{m}\right)$. Since $\bar{f}_{m}$ is degenerate along some edge of $\Gamma\left(\bar{f}_{m}\right)$, applying the first case to $\bar{f}_{m}$, we get $\mu\left(\bar{f}_{m}\right)>\mu_{N}\left(\bar{f}_{m}\right)$. Hence

$$
\mu(f)=\mu\left(\bar{f}_{m}\right)>\mu_{N}\left(\bar{f}_{m}\right) \geq \mu_{N}(f) .
$$

This proves Proposition 2.12.

Theorem 2.13. Let $f \in \mathfrak{m} \subset K[[x, y]]$ and let $f_{m}=f+x^{m}+y^{m}$. Then the following are equivalent 
(i) $\mu(f)=\mu_{N}(f)<\infty$.

(ii) $\mu(f)<\infty$ and $f_{m}$ is NND1 for some large integer number $m$.

(iii) $f$ is INND.

Proof. $(i) \Rightarrow(i i)$ : Since $\mu(f)=\mu_{N}(f)<\infty$ we have by definition of $\mu_{N}(f)$

$$
\mu\left(f_{m}\right)=\mu(f)=\mu_{N}(f)=\mu_{N}\left(f_{m}\right)<\infty .
$$

Combining Propositions 2.10, 2.11 and 2.12 we get the claim.

$($ ii $) \Rightarrow($ iii $)$ : Assume that $\mu(f)<\infty$ and $f_{m}$ is NND1. Firstly, it is easy to see that there is an $M \in \mathbb{N}$ such that $\Gamma(f) \subset \Gamma\left(f_{M}\right)$. It suffices to show $f$ is INND w.r.t. $\Gamma\left(f_{m}\right)$ for all $m>M$. We argue by contradiction. Suppose that it is not true. Then $f$ is not IND along some edge $\Delta$ of $\Gamma\left(f_{m}\right)$ which meets the axes, since $f_{m}$ is NND1. We may assume that $\Delta$ meets the axes at $(0, n)$. Let $(k, l)$ be the second vertex of $\Delta$. We consider two cases:

- If $l=0$, i.e. $\Gamma\left(f_{m}\right)$ has only one edge $\Delta$. Then $\Delta$ is also a unique edge of $\Gamma(f)$ and $\operatorname{in}_{\Delta}(f)=\operatorname{in}_{\Delta}\left(f_{m}\right)$. Since $f$ is not IND along $\Delta$, there exists $(a, b) \in$ $K \backslash\{(0,0)\}$ which is a zero point of $\mathrm{j}\left(\operatorname{in}_{\Delta}(f)\right)$. Beside, since $f_{m}$ is ND along $\Delta$, either $a=0$ or $b=0$. Assume that $a=0$ and $b \neq 0$. We will show that $f_{m}$ is not ND1 along $(0, n)$. Firstly, we write $\operatorname{in}_{\Delta}\left(f_{m}\right)=c_{0 n} y^{n}+x \cdot g(x, y)$, then $\frac{\partial \operatorname{in}_{\Delta}\left(f_{m}\right)}{\partial y}=n y^{n-1}+x \cdot \frac{\partial g}{\partial y}$. Thus

$$
\frac{\partial \operatorname{in}_{\Delta}\left(f_{m}\right)}{\partial y}(0, b)=n y^{n-1}=0 \Rightarrow p \neq 0 \text { and } p \mid n .
$$

We now write $\operatorname{in}_{\Delta}\left(f_{m}\right)=c_{0 n} y^{n}+c_{1 j} x y^{j}+x^{2} \cdot h(x, y)$, then

$$
\frac{\partial \operatorname{in}_{\Delta}\left(f_{m}\right)}{\partial x}=c_{1 j} y^{j}+2 x \cdot h(x, y)+x^{2} \cdot \frac{\partial h}{\partial x} .
$$

Since $\frac{\partial \operatorname{in}_{\Delta}\left(f_{m}\right)}{\partial x}(0, b)=0, c_{1 j}=0$. Hence $f_{m}$ is not ND1 along $(0, n)$, a contradiction.

- Assume that $l>0$. If $\Delta$ is also an edge of $\Gamma(f)$ then $\operatorname{in}_{\Delta}(f)=\operatorname{in}_{\Delta}\left(f_{m}\right)$. Since $f$ is not IND along $\Delta$, there exists $(a, b) \in K \times K^{*}$ being a zero of $\mathrm{j}\left(\operatorname{in}_{\Delta}(f)\right)$. Since $f_{m}$ is ND along $\Delta, a=0$. Analogously as above $f_{m}$ is not ND1 along $(0, n)$ and we get a contradiction. Assume now that $\Delta$ is not an edge of $\Gamma(f)$, i.e. $m=n$ and $x \mid f(x, y)$. Let $P$ be the end point of $\Gamma(f)$ closest to $y$-axis. It follows from $\Gamma(f) \subset \Gamma\left(f_{M}\right)$ and $m>M$ that $P$ must be a vertex of $\Delta$, i.e. $P=(k, l)$. This implies $f=x^{k} \cdot h(x, y)$. Since $\mu(f)<\infty, k=1$. Then $\operatorname{in}_{\Delta}(f)=c_{0 n} y^{n}+c_{1 l} x y^{l}$ and clearly $f$ is always IND along $\Delta$, a contradiction. Hence $f$ is INND w.r.t. $\Gamma\left(f_{m}\right)$ and then it is INND.

$($ iii $) \Rightarrow(i)$ : See Theorem 1.2 . 
Corollary 2.14. Let $f \in K[[x, y]]$ and let $M \in \mathbb{N}$ such that $\Gamma(f) \subset \Gamma\left(f_{M}\right)$. Then $f$ is INND if and only if it is INND w.r.t. $\Gamma\left(f_{m}\right)$ for some (equivalently for all) $m>M$.

Proof. One direction is obvious, it remains to show $f$ is INND $\Rightarrow f$ is INND w.r.t. $\Gamma\left(f_{m}\right)$ for all $m>M$. We take $m_{1}>M$ satisfying Theorem 2.13 and then

$f$ is INND $\Rightarrow \mu(f)<\infty$ and $f_{m_{1}}$ is NND1 $\Rightarrow f$ is INND w.r.t. $\Gamma\left(f_{m_{1}}\right)$.

For each inner face $\Delta_{m}$ of $\Gamma\left(f_{m}\right)$, since $m, m_{1}>M$, there is an inner face $\Delta_{m_{1}}$ of $\Gamma\left(f_{m_{1}}\right)$ such that $\operatorname{in}_{\Delta_{m}}(f)=\operatorname{in}_{\Delta_{m_{1}}}(f)$. Thus $f$ is IND along $\Delta_{m}$ since it is IND along $\Delta_{m_{1}}$. Hence $f$ is INND w.r.t. $\Gamma\left(f_{m}\right)$.

Corollary 2.15. Let $M \in \mathbb{N}$ be such that $\Gamma(f) \subset \Gamma\left(f_{M}\right)$. Then Theorem 2.13 holds for each $m>M$.

Remark 2.16. Let $\mu(f)<\infty$. Then $M$ can be chosen as the maximum of $n_{1}$ and $m_{1}$, where $n_{1}=n$ if $\Gamma(f) \cap\{x=0\}=\{(0, n)\}$ and $n_{1}=2 i_{1}$ if $\Gamma(f) \cap\{x=0\}=\emptyset$ and $\Gamma(f) \cap\{x=1\}=\left\{\left(1, i_{1}\right)\right\}$. Similarly we define $m_{1}$ with $x$ replaced by $y$. This remark and the previous corollaries are important for concrete computation.

Proof of Corollary 2.15. Clearly, the equivalence $(i) \Leftrightarrow($ iii $)$ does not depend on $m$ and as in the proof of Theorem 2.13 the implication $(i i) \Rightarrow($ iii $)$ holds for all $m>M$. It remains to show that $f$ is INND $\Rightarrow f_{m}$ is NND1. By Corollary 2.14, it suffices to show that $f$ is INND w.r.t. $\Gamma\left(f_{m}\right) \Rightarrow f_{m}$ is NND1. By contradiction, suppose that $f$ is INND w.r.t. $\Gamma\left(f_{m}\right)$ and $f_{m}$ is not NND1. Then $f$ is not ND1 along some vertex of $\Gamma\left(f_{m}\right)$ in the axes. Assume that $f$ is not ND1 along $(0, n) \in \Gamma\left(f_{m}\right)$. Then

$$
p \neq 0, p \mid n \text { and } \Gamma\left(f_{m}\right) \cap\{x=1\} \cap \operatorname{supp}\left(f_{m}\right)=\emptyset,
$$

i.e. $\left(f_{m}\right)_{\text {in }}=c_{0 n} y^{n}+x^{2} \cdot h(x, y)$. This implies $\mu\left(\left(f_{m}\right)_{\text {in }}\right)=\infty$. By Theorem 1.2 , $\left(f_{m}\right)_{i n}$ is not INND and then $f_{m}$ is also not INND, a contradiction.

Corollary 2.17. Let $K$ is a field of characteristic zero and $f \in \mathfrak{m} \subset K[[x, y]]$. Then the following are equivalent

(i) $\mu(f)=\mu_{N}(f)<\infty$.

(ii) $f$ is INND.

(iii) $f$ is $N N D$ and $\mu_{N}(f)<\infty$.

In particular, if $f$ is convenient then (i)-(iii) are equivalent to

(iv) $f$ is $N N D$.

Proof. The implications $(i) \Rightarrow(i i)$ and $(i i i) \Rightarrow(i)$ follow from Theorem 2.13 and Proposition 1.3. It remains to prove $(i i) \Rightarrow(i i i)$. 
Assume that $f$ is INND. Then by Theorem 2.13, $\mu_{N}(f)<\infty$. We will show that $f$ is ND along each vertex and each edge of $\Gamma(f)$. Since $\operatorname{char}(K)=0, f$ is ND along each vertex of $\Gamma(f)$. Let $\Delta$ be an edge of $\Gamma(f)$. Clearly, it is an inner edge of $\Gamma\left(f_{m}\right)$, where $m$ sufficiently large. Since $f$ is INND, by Corollary $2.14 f$ is INND w.r.t. $\Gamma\left(f_{m}\right)$. Then $f$ is IND along $\Delta$, and hence it is also ND along $\Delta$. This implies $f$ is NND.

Corollary 2.18. If $f$ is $N N D$ and $\mu_{N}(f)<\infty$ then $f$ is INND.

Proof. This follows from Proposition 1.3 and Theorem 2.13 .

Note that $\operatorname{char}(K)=0$ is only used to assure that $f$ is ND along each vertex of $\Gamma(f) \cap(\{0\} \times \mathbb{N} \cup \mathbb{N} \times\{0\})$. Hence, the last corollary holds also if $p>0$ and $p \not h$ if $(0, n)=\Gamma(f) \cap\{0\} \times \mathbb{N}$ and $p \not \supset m$ if $(m, 0)=\Gamma(f) \cap \mathbb{N} \times\{0\}$. Example 2.1 shows that this condition is necessary.

\section{3. $\delta$-INVARIANT}

We consider now another important invariant of plane curve singularities, the invariant $\delta$ and its combinatorial counterpart, the Newton invariant $\delta_{N}$. We show that both coincide iff $f$ is weighted homogeneous Newton non-degenerate (WHNND), a new non-degenerate condition introduced below.

Let $f \in \mathfrak{m} \subset K[[x, y]]$ be a power series. We define the multiplicity of $f$, denoted by $\operatorname{mt}(f)$, to be the minimal degree of the homogeneous part of $f$. So

$$
f=\sum_{k \geq m:=\operatorname{mt}(f)} f_{k}(x, y),
$$

where $f_{k}$ is homogeneous of degree $k$ and $f_{m} \neq 0$. Then $f_{m}$ decomposes into linear factors,

$$
f_{m}=\prod_{i=1}^{s}\left(\alpha_{i} x-\beta_{i} y\right)^{r_{i}},
$$

with $\left(\beta_{i}: \alpha_{i}\right) \in \mathbb{P}^{1}$ pairwise distinct. We call $f_{m}$ the tangent cone and the points $\left(\beta_{i}: \alpha_{i}\right), i=1, \ldots, s$, the tangent directions of $f$.

We fix a minimal resolution of the singularity computed via successively blowing up points, denote by $Q \rightarrow 0$ that $Q$ is an infinitely near point of the origin on $f$. If $Q$ is an infinitely near point in the $n$-th neighbourhood of 0 , we denote by $m_{Q}$ the multiplicity of the $n$-th strict transform of $f$ at $Q$. If $P$ is an infinitely near point in the $l$-th neighbourhood of 0 , we denote by $Q \rightarrow P$ that $Q$ is also an infinitely near point of $P$ on the $l$-th strict transform $\tilde{f}_{l}$ of $f$ at $P$. Note that if $Q \rightarrow P$ then $n \geq l$ and we set $n\left(\tilde{f}_{l}, Q\right):=n-l$. In particular, we have $n(f, Q)=n$. 
Let $E_{1}, \ldots, E_{k}$ be the edges of the Newton diagram of $f$. We denote by $l\left(E_{i}\right)$ the lattice length of $E_{i}$, i.e. the number of lattice points on $E_{i}$ minus one and by $s\left(f_{E_{i}}\right)$ the number of non-monomial irreducible (reduced) factors of $f_{E_{i}}$. We set

(a) $\delta(f):=\sum_{Q \rightarrow 0} \frac{m_{Q}\left(m_{Q}-1\right)}{2}$ the delta invariant of $f$. The delta invariant $\delta(f)$ equals also $\operatorname{dim}_{K}(\bar{R} / R)$ where $R=K[[x, y]] /\langle f\rangle$ and $\bar{R}$ is the integral closure of of $R$ in its total ring of fractions.

(b) $\nu(f):=\sum_{Q \text { special }} \frac{m_{Q}\left(m_{Q}-1\right)}{2}$, where an infinitely near point $Q$ is special if it is the origin or the origin of the corresponding chart of the blowing up.

(c) $r(f)$ the number of branches of $f$ counted with multiplicity.

(d) If $f$ is convenient, we define

$$
\delta_{N}(f):=V_{2}\left(\Gamma_{-}(f)\right)-\frac{V_{1}\left(\Gamma_{-}(f)\right)}{2}+\frac{\sum_{i=1}^{k} l\left(E_{i}\right)}{2},
$$

and otherwise we set $\delta_{N}(f):=\sup \left\{\delta_{N}\left(f^{(m)}\right) \mid f^{(m)}:=f+x^{m}+y^{m}, m \in \mathbb{N}\right\}$ and call it the Newton $\delta$-invariant of $f$.

(e) $r_{N}(f):=\sum_{i=1}^{k} l\left(E_{i}\right)+\max \left\{j \mid x^{j}\right.$ divides $\left.f\right\}+\max \left\{l \mid y^{l}\right.$ divides $\left.f\right\}$.

(f) $s_{N}(f):=\sum_{i=1}^{k} s\left(f_{E_{i}}\right)+\max \left\{j \mid x^{j}\right.$ divides $\left.f\right\}+\max \left\{l \mid y^{l}\right.$ divides $\left.f\right\}$.

Note that $\delta(f)$ and $r(f)$ are coordinate-independent while all the other ones depend (only) on the Newton diagram of $f$ and hence are coordinate-dependent (for $\nu(f)$ see Proposition 3.9).

Proposition 3.1. For $0 \neq f \in\langle x, y\rangle$ we have $r(f) \leq r_{N}(f)$, and if $f$ is WNND then $r(f)=r_{N}(f)$.

Proof. cf. [BGM10, Lemma 4.10]

Let $E$ be an edge of the Newton diagram of $f$. Then we can write $f_{E}$ as follows,

$$
f_{E}=\text { monomial } \times \prod_{i=1}^{s}\left(a_{i} x^{m_{0}}-b_{i} y^{n_{0}}\right)^{r_{i}},
$$

where $a_{i}, b_{i} \in K^{*},\left(a_{i}: b_{i}\right)$ pairwise distinct; $m_{0}, n_{0}, r_{i} \in \mathbb{N}_{>0}, \operatorname{gcd}\left(m_{0}, n_{0}\right)=1$. It easy to see that

$$
s=s\left(f_{E}\right) \text { and } l(E)=\sum_{i=1}^{s} r_{i} .
$$

This implies $s\left(f_{E}\right) \leq l(E)$ and hence $s_{N}(f) \leq r_{N}(f)$.

Let $f=f_{d}^{\mathrm{w}}+f_{d+1}^{\mathrm{w}}+\ldots$ with $f_{d}^{\mathrm{w}} \neq 0$ be the $\left(n_{0}, m_{0}\right)$-weighted homogeneous decomposition of $f$.

Definition 3.2. We say that $f$ is weighted homogeneous non-degenerate (WHND) along $E$ if either $r_{i}=1$ for all $i=1, \ldots, s$ or $\left(a_{i} x^{m_{0}}-b_{i} y^{n_{0}}\right)$ does not divide $f_{d+1}^{\mathrm{w}}$ for each $r_{i}>1$.

$f$ is called weighted homogeneous Newton non-degenerate (WHNND) if its Newton diagram has no edge or if it is WHND along each edge of its Newton diagram. 
Lemma 3.3. Let $f \in K[[x, y]]$. Then $f$ is not WHNND if and only if there exist $a, b \in K^{*}, m, n \in \mathbb{N}_{>0}$ with $(m, n)=1$ such that $f_{d}^{\mathrm{w}}$ is divisible by $\left(a x^{m}-b y^{n}\right)^{2}$ and $f_{d+1}^{\mathrm{w}}$ is divisible by $\left(a x^{m}-b y^{n}\right)$, where $f_{d}^{\mathrm{w}}$ (resp. $\left.f_{d+1}^{\mathrm{w}}\right)$ is the first (resp. the second) term of the $(n, m)$-weighted decomposition of $f$.

Proof. Straightforward from the above definition.

Remark 3.4. (a) In [Lu87] the author introduced superisolated singularities to study the $\mu$-constant stratum. We recall that $f \in K[[x, y]]$ is superisolated if it becomes regular after only one blowing up. By ([Lu87, Lemma 1]), this is equivalent to: $f_{m+1}\left(\beta_{i}, \alpha_{i}\right) \neq 0$ for all tangent directions $\left(\beta_{i}: \alpha_{i}\right)$ of $f$ with $r_{i}>1$, where $f=f_{m}+f_{m+1}+\ldots$ is the homogeneous decomposition of $f$ and

$$
f_{m}=\prod_{i=1}^{s}\left(\alpha_{i} x-\beta_{i} y\right)^{r_{i}} .
$$

Note that this condition concerns all factors of $f_{m}$ including monomials. For WHNND singularities we require a similar condition, but for "all weights" and without any condition on the monomial factors of the first term of the weigted homogeneous decomposition of $f$.

(b) Since a plane curve singularity is superisolated iff it becomes regular after only one blowing up, we have $\delta(f)=\nu(f)=m(m-1) / 2$ and hence $\delta(f)=$ $\delta_{N}(f)=m(m-1) / 2$, by Proposition 3.9. It follows from Theorem 3.12 that

(c) A superisolated plane curve singularity is WHNND.

(d) The plane curve singularity $x^{2}+y^{5}$ is WHNND but not superisolated.

Proposition 3.5. With notations as above, $f$ is WND along $E$ if and only if $s\left(f_{E}\right)=l(E)$ or, equivalently, iff $r_{i}=1$ for all $i=1, \ldots, s$. In particular, WNND implies WHNND.

Proof. Firstly we can see that the equation $s\left(f_{E}\right)=l(E)$ is equivalent to $r_{i}=1$ for all $i=1, \ldots, s$ since $s\left(f_{E}\right)=s$ and $l(E)=r_{1}+\ldots+r_{s}$. It remains to prove that $f$ is WND along $E$ iff $r_{i}=1$ for all $i=1, \ldots, s$. Assume that there is an $i_{0}$ s.t. $r_{i_{0}}>1$. It is easy to see that $f_{E}, \frac{\partial f_{E}}{\partial x}, \frac{\partial f_{E}}{\partial y}$ are divisible by $\left(a_{i_{0}} x_{0}^{m_{0}}-b_{i_{0}} y_{0}^{n_{0}}\right)$. Hence $f$ is weakly degenerate (WD) along $E$.

We now assume that $f$ is weakly degenerate (WD) along $E$. Then there exist $x_{0}, y_{0} \in K^{*}$ such that

$$
f_{E}\left(x_{0}, y_{0}\right)=\frac{\partial f_{E}}{\partial x}\left(x_{0}, y_{0}\right)=\frac{f_{E}}{\partial y}\left(x_{0}, y_{0}\right)=0
$$

and hence there exists an index $i_{0}$ such that $a_{i_{0}} x_{0}^{m_{0}}-b_{i_{0}} y_{0}^{n_{0}}=0$. We will show that $r_{i_{0}}>1$. In fact, if this is not true then $f_{E}(x, y)=\left(a_{i_{0}} x^{m_{0}}-b_{i_{0}} y^{n_{0}}\right) \cdot h(x, y)$ 
with $h\left(x_{0}, y_{0}\right) \neq 0$. Since

$$
\frac{\partial f_{E}}{\partial x}\left(x_{0}, y_{0}\right)=\frac{f_{E}}{\partial y}\left(x_{0}, y_{0}\right)=0
$$

this is impossible if $p=0$ and implies that $p$ divides $m_{0}$ and $n_{0}$ if $p>0$. This contradicts the assumption $\operatorname{gcd}\left(m_{0}, n_{0}\right)=1$.

Let $f \in K[[x, y]]$ and let $E_{i}, i=1, \ldots, k$ be the edges of its Newton diagram. Then by Proposition 2.6 there is a factorization of $f$,

$$
f=\text { monomial } \cdot \bar{f}_{1} \cdot \ldots \cdot \bar{f}_{k}
$$

such that $\bar{f}_{i}$ is convenient and $f_{E_{i}}=$ monomial $\times\left(\bar{f}_{i}\right)_{i n}$. Note that $\bar{f}_{i}$ is in general not irreducible. On the other hand, $f$ can be factorized into its irreducible factors as $f=m_{1} \cdot \ldots \cdot m_{l} \cdot f_{1} \cdot \ldots \cdot f_{r}$, where $m_{j}$ are monomials, and $f_{j}$ are convenient.

Proposition 3.6. (a) Let $g, h \in K[[x, y]]$ such that $f=g \cdot h$. If $f$ is WHNND then $g$ and $h$ are also WHNND.

(b) With the above notations, the following are equivalent:

(i) $f$ is WHNND.

(ii) $\bar{f}_{1}, \ldots, \bar{f}_{k}$ are WHNND.

(iii) $f_{1}, \ldots, f_{r}$ are $W H N N D$ and $\left(f_{i}\right)_{\text {in }}$ are pairwise coprime.

Proof. (a) It suffice to show that if $g$ is not WHNND then neither is $f$. In fact, since $g$ is not WHNND, by Lemma 3.3, there exist $a, b \in K^{*}, m, n \in \mathbb{N}_{>0}$ with $(m, n)=1$ such that $g_{c}^{\mathrm{w}}$ is divisible by $\left(a x^{m}-b y^{n}\right)^{2}$ and $g_{c+1}^{\mathrm{w}}$ is divisible by $\left(a x^{m}-b y^{n}\right)$, where $g_{c}^{\mathrm{w}}$ (resp. $\left.g_{c+1}^{\mathrm{w}}\right)$ is the first (resp. the second) term of the $(n, m)$-weighted decomposition of $g$. Let $f=f_{d}^{\mathrm{w}}+f_{d+1}^{\mathrm{w}}+\ldots$ (resp. $h=$ $\left.h_{e}^{\mathrm{w}}+h_{e+1}^{\mathrm{w}}+\ldots\right)$ be the $(n, m)$-weighted homogeneous decomposition of $f$ (resp. h). Then

$$
f_{d}^{\mathrm{w}}=g_{c}^{\mathrm{w}} \cdot h_{e}^{\mathrm{w}} \text { and } f_{d+1}^{\mathrm{w}}=g_{c}^{\mathrm{w}} \cdot h_{e+1}^{\mathrm{w}}+g_{c+1}^{\mathrm{w}} \cdot h_{e}^{\mathrm{w}} .
$$

This implies that $f_{d}^{\mathrm{w}}$ is divisible by $\left(a x^{m}-b y^{n}\right)^{2}$ and $f_{d+1}^{\mathrm{w}}$ is divisible by $\left(a x^{m}-\right.$ $\left.b y^{n}\right)$. Again by Lemma 3.3, $f$ is not WHNND.

(b) It is easily verified that we may restrict to the case that $f$ is convenient.

The implication (i) $\Rightarrow$ (ii) follows from part (a).

(ii) $\Rightarrow$ (iii): Assume that $\bar{f}_{1}, \ldots, \bar{f}_{k}$ are WHNND. By part (a) we can deduce that $f_{1}, \ldots, f_{r}$ are WHNND since for each $i, f_{i}$ is an irreducible factor of some $\bar{f}_{j}$. We now show that the $\left(f_{i}\right)_{i n}$ are pairwise coprime. By contradiction, suppose that $\left(f_{1}\right)_{\text {in }}$ and $\left(f_{2}\right)_{\text {in }}$ are not coprime. It follows from Proposition 2.5 that there exist $a, b \in K^{*}, m, n \in \mathbb{N}_{>0}$ with $(m, n)=1$ such that $\left(a x^{m}-b y^{n}\right)$ is the unique irreducible factor of $\left(f_{1}\right)_{\text {in }}$ and $\left(f_{2}\right)_{i n}$. Consequently, $\left(f_{1}\right)_{\text {in }}$ and $\left(f_{2}\right)_{\text {in }}$ are both $(n, m)$-weighted homogeneous. Assume that $f_{1}$ resp. $f_{2}$ is an irreducible factor 
of $\bar{f}_{j_{1}}$ resp. $\bar{f}_{j_{2}}$ for some $j_{1}$ and $j_{2}$. Since $\left(\bar{f}_{j_{1}}\right)_{i n}$ and $\left(\bar{f}_{j_{2}}\right)_{i n}$ are weighted homogeneous, $\left(f_{1}\right)_{i n}$ resp. $\left(f_{2}\right)_{i n}$ is a factor of $\left(\bar{f}_{j_{1}}\right)_{i n}$ resp. $\left(\bar{f}_{j_{2}}\right)_{i n}$. This implies that $\left(\bar{f}_{j_{1}}\right)_{i n}$ and $\left(\bar{f}_{j_{2}}\right)_{i n}$ and therefore $f_{E_{j_{1}}}$ and $f_{E_{j_{2}}}$ are all $(n, m)$-weighted homogeneous. Then the edge $E_{j_{1}}$ must coincide the edge $E_{j_{2}}$ and hence $\bar{f}_{j_{1}}=\bar{f}_{j_{2}}$. It yields that the product $g:=f_{1} \cdot f_{2}$ is a factor of $\bar{f}_{j_{1}}$. Now, we decompose $g, f_{1}, f_{2}$ into their $(n, m)$-weighted homogeneous terms as follows:

$$
g=g_{c}^{\mathrm{w}}+g_{c+1}^{\mathrm{w}}+\ldots, f_{1}=\left(f_{1}\right)_{d_{1}}^{\mathrm{w}}+\left(f_{1}\right)_{d_{1}+1}^{\mathrm{w}}+\ldots, f_{2}=\left(f_{2}\right)_{d_{2}}^{\mathrm{w}}+\left(f_{2}\right)_{d_{2}+1}^{\mathrm{w}}+\ldots
$$

Then $c=d_{1}+d_{2},\left(f_{1}\right)_{d_{1}}^{\mathrm{w}}=\left(f_{1}\right)_{i n},\left(f_{2}\right)_{d_{2}}^{\mathrm{w}}=\left(f_{2}\right)_{i n}, g_{c}^{\mathrm{w}}=\left(f_{1}\right)_{d_{1}}^{\mathrm{w}} \cdot\left(f_{2}\right)_{d_{2}}^{\mathrm{w}}$ and $g_{c+1}^{\mathrm{w}}=\left(f_{1}\right)_{d_{1}}^{\mathrm{w}} \cdot\left(f_{2}\right)_{d_{2}+1}^{\mathrm{w}}+\left(f_{1}\right)_{d_{1}+1}^{\mathrm{w}} \cdot\left(f_{2}\right)_{d_{2}}^{\mathrm{w}}$. This implies that $g_{c}^{\mathrm{w}}$ is divisible by $\left(a x^{m}-b y^{n}\right)^{2}$ and $g_{c+1}^{\mathrm{w}}$ is divisible by $\left(a x^{m}-b y^{n}\right)$. It follows from Lemma 3.3 that $g$ is not WHNND and hence $\bar{f}_{j_{1}}$ is also not WHNND by part (a) with $g$ a factor of $\bar{f}_{j_{1}}$, which is a contradiction.

(iii) $\Rightarrow$ (i): Suppose that $f$ is not WHNND and that the $\left(f_{i}\right)_{i n}$ are pairwise coprime. We will show that $f_{i}$ is not WHNND for some $i$. Indeed, since $f$ is not WHNND, by Lemma 3.3, there exist $a, b \in K^{*}, m, n \in \mathbb{N}_{>0}$ with $(m, n)=1$ such that $f_{d}^{\mathrm{w}}$ is divisible by $\left(a x^{m}-b y^{n}\right)^{2}$ and $f_{d+1}^{\mathrm{w}}$ is divisible by $\left(a x^{m}-b y^{n}\right)$, where $f_{d}^{\mathrm{w}}$ (resp. $f_{d+1}^{\mathrm{w}}$ ) is the first (resp. the second) term of the $(n, m)$-weighted decomposition of $f$. Let $\left(f_{i}\right)=\left(f_{i}\right)_{d_{i}}^{\mathrm{w}}+\left(f_{i}\right)_{d_{i}+1}^{\mathrm{w}}+\ldots$ be the $(n, m)$-weighted homogeneous decomposition of $f_{i}, i=1, \ldots, r$. Then we have

$$
d=\sum_{i=1}^{r} d_{i} ; \quad\left(f_{i}\right)_{d_{i}}^{\mathrm{w}}=\left(f_{i}\right)_{i n} ; \quad f_{d}^{\mathrm{w}}=\prod_{i=1}^{r}\left(f_{i}\right)_{d_{i}}^{\mathrm{w}} ; \quad f_{d+1}^{\mathrm{w}}=\sum_{i=1}^{r}\left(\left(f_{i}\right)_{d_{i}+1}^{\mathrm{w}} \cdot \prod_{l \neq i}\left(f_{l}\right)_{d_{l}}^{\mathrm{w}}\right)
$$

Since $f_{d}^{\mathrm{w}}=\prod_{i=1}^{r}\left(f_{i}\right)_{d_{i}}^{\mathrm{w}}$ and since the $\left(f_{i}\right)_{d_{i}}^{\mathrm{w}}$ are pairwise coprime, there exists an $i_{0}$ such that $\left(f_{i_{0}}\right)_{d_{i_{0}}}^{\mathrm{w}}$ is divisible by $\left(a x^{m}-b y^{n}\right)^{2}$ and $\left(f_{l}\right)_{d_{l}}^{\mathrm{w}}$ is not divisible by $\left(a x^{m}-b y^{n}\right)$ for all $l \neq i_{0}$. This implies that $\left(f_{i_{0}}\right)_{d_{i_{0}}+1}^{\mathrm{w}}$ is divisible by $\left(a x^{m}-b y^{n}\right)$ since

$$
f_{d+1}^{\mathrm{w}}=\left(f_{i_{0}}\right)_{d_{i_{0}}+1}^{\mathrm{w}} \cdot \prod_{l \neq i_{0}}\left(f_{l}\right)_{d_{l}}^{\mathrm{w}}+\sum_{i \neq i_{0}}\left(\left(f_{i}\right)_{d_{i}+1}^{\mathrm{w}} \cdot \prod_{l \neq i}\left(f_{l}\right)_{d_{l}}^{\mathrm{w}}\right) .
$$

Then $f_{i_{0}}$ is not WHNND by Lemma 3.3 .

Proposition 3.7. For $0 \neq f \in\langle x, y\rangle$ we have $s_{N}(f) \leq r(f)$ and if $f$ is WHNND then $s_{N}(f)=r(f)$.

Proof. If $f=x^{j} y^{l} \cdot g(x, y)$ with $g$ convenient, then

$$
s_{N}(f)=s_{N}(g)+j+l \text { and } r(f)=r(g)+j+l,
$$

so we may assume that $f$ is convenient.

Step 1. Assume first that the Newton diagram $\Gamma(f)$ has only one edge $E$. Then we can see that $f_{i n}=\prod_{i=1}^{r}\left(f_{i}\right)_{i n}$. It follows from Proposition 2.5 that for each $i$, 
$\left(f_{i}\right)_{\text {in }}$ has only one irreducible factor and therefore $f_{\text {in }}$ has at most $r$ irreducible factors. This means that $r \geq s_{N}(f)$.

If $r(f)>s_{N}(f)$, then there exist $i \neq j$ such that $\left(f_{i}\right)_{i n}$ and $\left(f_{j}\right)_{i n}$ have the same factor. This means that $\left(f_{i}\right)_{i n}$ and $\left(f_{j}\right)_{i n}$ are not coprime. Then by Proposition 3.6, $f$ is not WHNND.

Step 2. Assume now that the Newton diagram $\Gamma(f)$ has $k$ edges $E_{1}, \ldots, E_{k}$. By Proposition 2.6, $f$ can be factorized as $f=\bar{f}_{1} \cdot \ldots \cdot \bar{f}_{k}$, where $\bar{f}_{j}$ is convenient, its Newton diagram has only one edge and $f_{E_{j}}=$ monomial $\cdot\left(\bar{f}_{j}\right)_{i n}$ for each $j=1, \ldots, k$. This implies that $s_{N}\left(\bar{f}_{j}\right)=s\left(f_{E_{j}}\right)$. Then we obtain

$$
r(f)=\sum_{j=1}^{k} r\left(\bar{f}_{j}\right) \geq \sum_{j=1}^{k} s_{N}\left(\bar{f}_{j}\right)=\sum_{j=1}^{k} s\left(f_{E_{j}}\right)=s_{N}(f) .
$$

Now we assume that $r(f)>s_{N}(f)$. Then there exists a $j=1, \ldots, k$ such that $r\left(\bar{f}_{j}\right)>s\left(f_{E_{j}}\right)=s_{N}\left(\bar{f}_{j}\right)$. It follows from Step 1 that $\bar{f}_{j}$ is not WHNND. Hence $f$ is not WHNND by Proposition 3.6, which proves the proposition.

Proposition 3.8. For $0 \neq f \in\langle x, y\rangle$ we have $s_{N}(f) \leq r(f) \leq r_{N}(f)$, and both equalities hold if and only if $f$ is WNND.

Proof. The inequalities follow from Proposition 3.1 and Proposition 3.7, For each edge $E$ of $\Gamma(f)$, by Proposition 3.5, $f$ is WND along $E$ iff $s\left(f_{E}\right)=l(E)$. This implies that $f$ is WNND if and only if $s_{N}(f)=r_{N}(f)$ since $s\left(f_{E}\right) \leq l(E)$ and both sides are additive with respect to edges of $\Gamma(f)$.

We investigate now the relations between $\nu(f), \delta_{N}(f)$ and $\delta(f)$, which were studied in [BeP00] and [BGM10].

Proposition 3.9. BGM10, Lemma 4.8] If $f \in K[[x, y]]$ then $\delta_{N}(f)=\nu(f)$.

Proposition 3.10. BGM10, Prop. 4.9] For $0 \neq f \in\langle x, y\rangle$ we have $\delta_{N}(f) \leq$ $\delta(f)$, and if $f$ is $W N N D$ then $\delta_{N}(f)=\delta(f)$.

Hence WNND is sufficient but, by the following example, not necessary for $\delta_{N}(f)=\delta(f)$.

Example 3.11. Let $f(x, y)=(x+y)^{2}+y^{3} \in K[[x, y]]$. Then $f$ is not WNND but $\delta_{N}(f)=\delta(f)=1$. This easy example shows also that WNND depends on the coordinates since $x^{2}+y^{3}$ is WNND. Note that $f$ is WHNND.

Now we prove that WHNND is necessary and sufficient for $\delta_{N}(f)=\delta(f)$.

Theorem 3.12. Let $f \in K[[x, y]]$ be reduced. Then $\delta(f)=\delta_{N}(f)$ if and only if $f$ is WHNND. 
We will prove the theorem after three technical lemmas.

Let $E$ be an edge of the Newton diagram of $f$. We write

$$
f_{E}=\text { monomial } \times \prod_{i=1}^{s}\left(a_{i} x^{m_{0}}-b_{i} y^{n_{0}}\right)^{r_{i}},
$$

where $a_{i}, b_{i} \in K^{*},\left(a_{i}: b_{i}\right)$ pairwise distinct; $m_{0}, n_{0}, r_{i} \in \mathbb{N}_{>0},\left(m_{0}, n_{0}\right)=1$.

Lemma 3.13. With the above notations, there exist an integer $n$ and an infinitely near point $P_{n}$ in the $n$-th neighbourhood of 0 , such that

$$
\left(\tilde{f}_{n}\right)_{E_{n}}(u, v)=\text { monomial } \times \prod_{i=1}^{s}\left(a_{i} u-b_{i} v\right)^{r_{i}},
$$

where $\tilde{f}_{n}$ is a local equation of the strict transform of $\tilde{f}$ at $P_{n}$ and $E_{n}$ is some edge of its Newton diagram $\Gamma\left(\tilde{f}_{n}\right)$. Moreover, $f$ is WHND along $E$ if and only if $\tilde{f}_{n}$ is WHND along $E_{n}$.

Proof. We prove the lemma by induction on $m_{0}+n_{0}$. If $m_{0}+n_{0}=2$, i.e. $m_{0}=n_{0}=1$, then the claim is trivial.

Suppose $m_{0}+n_{0}>2$. Now we show the induction step. Since $m_{0}+n_{0}>2$ and $\operatorname{gcd}\left(m_{0}, n_{0}\right)=1, m_{0} \neq n_{0}$. We may then assume that $m_{0}<n_{0}$. Then $P_{1}:=(1,0)$ is a special infinitely near point of 0 and the local equation of $\tilde{f}_{1}$ at $P_{1}$ in chart 2 , is:

$$
\tilde{f}_{1}\left(x_{1}, y_{1}\right)=\frac{f\left(x_{1} y_{1}, y_{1}\right)}{y_{1}^{m}}, \text { where } m=\operatorname{mt}(f) .
$$

Let $f=f_{d_{0}}^{\mathrm{w}}+f_{d_{0}+1}^{\mathrm{w}}+\ldots$ with $f_{d_{0}}^{\mathrm{w}} \neq 0$ be the $\left(n_{0}, m_{0}\right)$-weighted homogeneous decomposition of $f$. It is easy to see that $\tilde{f}_{1}=\left(\tilde{f}_{1}\right)_{e_{0}}^{\mathrm{w}}+\left(\tilde{f}_{1}\right)_{e_{0}+1}^{\mathrm{w}}+\ldots$ is the $\left(n_{0}-m_{0}, m_{0}\right)$-weighted homogeneous decomposition of $\tilde{f}_{1}$ with

$$
e_{0}=d_{0}-m \cdot m_{0} \text { and }\left(\tilde{f}_{1}\right)_{e_{0}+\nu}^{\mathrm{w}}=\frac{f_{d_{0}+\nu}^{\mathrm{w}}\left(x_{1} y_{1}, y_{1}\right)}{y_{1}^{m}}, \forall \nu \geq 0 .
$$

In particular,

$$
\left(\tilde{f}_{1}\right)_{e_{0}}^{\mathrm{w}}=\text { monomial } \times \prod_{i=1}^{s}\left(a_{i} x^{m_{0}}-b_{i} y^{n_{0}-m_{0}}\right)^{r_{i}} .
$$

We denote by $E_{1}$ the convex hull of the support of $\left(\tilde{f}_{1}\right)_{e_{0}}^{\mathrm{w}}$. Clearly, $E_{1}$ is an edge of $\Gamma\left(\tilde{f}_{1}\right)$. Since

$$
\left(\tilde{f}_{1}\right)_{E_{1}}=\left(\tilde{f}_{1}\right)_{e_{0}}^{\mathrm{w}}=\text { monomial } \times \prod_{i=1}^{s}\left(a_{i} x^{m_{0}}-b_{i} y^{n_{0}-m_{0}}\right)^{r_{i}}
$$

and since $\left(\tilde{f}_{1}\right)_{e_{0}+1}^{\mathrm{w}}=\frac{f_{d_{0}+1}^{\mathrm{w}}\left(x_{1} y_{1}, y_{1}\right)}{y_{1}^{m}}$, it follows that $f$ is WHND along $E$ iff $\tilde{f}_{1}$ is also WHND along $E_{1}$. Hence the induction step is proven by applying the induction hypothesis to $\tilde{f}_{1}$. 
The above lemma yields that $Q_{E, i}:=\left(b_{i}: a_{i}\right), i=1, \ldots, s$, are determined by $f_{E}$ and they are tangent directions of $\tilde{f}_{n}$. Then they are infinitely near points in the first neighbourhood of $P_{n}$. Consequently, they are infinitely near points in the $(n+1)$-th neighbourhood of 0 . To compute the multiplicity $m_{Q_{E, i}}$, we consider the local equation of the strict transform $\tilde{f}_{n+1}$ of $\tilde{f}_{n}$ at $Q_{E, i}=\left(b_{i}: a_{i}\right)$ in chart 2:

$$
\begin{aligned}
\tilde{f}_{n+1}\left(u_{1}, v_{1}\right) & =\frac{\tilde{f}_{n}\left(\left(u_{1}+\frac{b_{i}}{a_{i}}\right) v_{1}, v_{1}\right)}{v_{1}^{e_{0}}} \\
& =\frac{\left(\tilde{f}_{n}\right)_{e_{0}}^{\mathrm{w}}\left(\left(u_{1}+\frac{b_{i}}{a_{i}}\right) v_{1}, v_{1}\right)}{v_{1}^{e_{0}}}+\frac{\left(\tilde{f}_{n}\right)_{e_{0}+1}^{\mathrm{w}}\left(\left(u_{1}+\frac{b_{i}}{a_{i}}\right) v_{1}, v_{1}\right)}{v_{1}^{e_{0}}}+\ldots \\
& =\left(\tilde{f}_{n}\right)_{e_{0}}^{\mathrm{w}}\left(u_{1}+\frac{b_{i}}{a_{i}}, 1\right)+v_{1} \cdot\left(\tilde{f}_{n}\right)_{e_{0}+1}^{\mathrm{w}}\left(u_{1}+\frac{b_{i}}{a_{i}}, 1\right)+\ldots,
\end{aligned}
$$

where $\tilde{f}_{n}=\left(\tilde{f}_{n}\right)_{e_{0}}^{\mathrm{w}}+\left(\tilde{f}_{n}\right)_{e_{0}+1}^{\mathrm{w}}+\ldots$ with $\left(\tilde{f}_{n}\right)_{e_{0}}^{\mathrm{w}} \neq 0$, is the $((1,1)$-weighted $)$ homogeneous decomposition of $f$. Since

$$
\left(\tilde{f}_{n}\right)_{E_{n}}(u, v)=\left(\tilde{f}_{n}\right)_{e_{0}}^{\mathrm{w}}(u, v)=\left(a_{i} u-b_{i} v\right)^{r_{i}} \cdot g(u, v) \text { with } g\left(b_{i}, a_{i}\right) \neq 0,
$$

we get

$$
\tilde{f}_{n+1}\left(u_{1}, v_{1}\right)=\left(a_{i} u_{1}\right)^{r_{i}} \cdot g\left(u_{1}+\frac{b_{i}}{a_{i}}, 1\right)+v_{1} \cdot\left(\tilde{f}_{n}\right)_{e_{0}+1}^{\mathrm{w}}\left(u_{1}+\frac{b_{i}}{a_{i}}, 1\right)+\ldots,
$$

with $g\left(u_{1}+\frac{b_{i}}{a_{i}}, 1\right)$ a unit. In the following, this equality will be used to compare the multiplicity $\operatorname{mt}\left(\tilde{f}_{n+1}\right)$ with 1 .

Lemma 3.14. With the above notations,

(a) if $f$ is WHND along $E$, then $m_{Q_{E, i}}=1$ for all $i$;

(b) if $f$ is not WHND along $E$, then $m_{Q_{E, i}}>1$ for some $i$.

Proof. (a) Since $f$ is WHND along $E$, it follows from Lemma 3.13 that $f_{n}$ is WHND along $E_{n}$, i.e. either $r_{i}=1$ for all $i$ or $\left(a_{i} u-b_{i} v\right)$ is not a factor of $\left(\tilde{f}_{n}\right)_{e_{0}+1}^{\mathrm{w}}$ for each $r_{i}>1$. If $r_{i}=1$, it is easy to see that $m_{Q_{E, i}}=\operatorname{mt}\left(\tilde{f}_{n+1}\left(u_{1}, v_{1}\right)\right)=1$ for all $i$. If $r_{i}>1$ and $\left(a_{i} u-b_{i} v\right)$ is not a factor of $\left(\tilde{f}_{n}\right)_{e_{0}+1}^{\mathrm{w}}$. Then $\left(\tilde{f}_{n}\right)_{e_{0}+1}^{\mathrm{w}}\left(b_{i}, a_{i}\right) \neq 0$. This implies that $\left(\tilde{f}_{n}\right)_{e_{0}+1}^{\mathrm{w}}\left(u_{1}+\frac{b_{i}}{a_{i}}, 1\right)$ is a unit. Hence

$$
m_{Q_{E, i}}=\operatorname{mt}\left(\tilde{f}_{n+1}\left(u_{1}, v_{1}\right)\right)=1 .
$$

(b) Assume that $f$ is not WHND along $E$. By Lemma 3.13, $\tilde{f}_{n}$ is not WHND along $E_{n}$, i.e. there exists an $i$ such that $r_{i}>1$ and $\left(a_{i} u-b_{i} v\right)$ is a factor of $\left(\tilde{f}_{n}\right)_{e_{0}+1}^{\mathrm{w}}$. Therefore $\left(\tilde{f}_{n}\right)_{e_{0}+1}^{\mathrm{w}}(u, v)=\left(a_{i} u-b_{i} v\right) \cdot h(u, v)$ and then

$$
\left(\tilde{f}_{n}\right)_{e_{0}+1}^{\mathrm{w}}\left(u_{1}+\frac{b_{i}}{a_{i}}, 1\right)=\left(a_{i} u_{1}\right) \cdot h\left(u_{1}+\frac{b_{i}}{a_{i}}, 1\right) .
$$

Hence $m_{Q_{E, i}}=\operatorname{mt}\left(\tilde{f}_{n+1}\left(u_{1}, v_{1}\right)\right)>1$. 
Lemma 3.15. With the above notations, if $Q$ is not special, then there exists an edge $E$ of $\Gamma(f)$ such that $Q \rightarrow Q_{E, i}$ for some $i$.

Proof. We will prove the lemma by induction on $n(f, Q)$. First, since $Q$ is not special, $n(f, Q) \geq 1$. If $n(f, Q)=1$, then $Q$ is a tangent direction of $f$ and we can write $Q=(b: a)$, where $(a x-b y)$ is a factor of the tangent cone $f_{m}$ of $f$. Since $Q$ is not special, $f_{m}$ is not monomial. This implies that there exists an edge $E$ of $\Gamma(f)$ such that $f_{E}=f_{m}$. We can write

$$
f_{E}=f_{m}=\text { monomial } \times \prod_{i=1}^{s}\left(a_{i} x-b_{i} y\right)^{r_{i}}
$$

with $(b: a)=\left(b_{1}: a_{1}\right)$, consequently $Q=Q_{E, 1}$.

Now we prove the induction step. Suppose that $n(f, Q)>1$. Then $Q \rightarrow P$ for some infinitely near point $P$ in the first neighbourhood of 0 . If $P$ is not special, then as above, $P=Q_{E, 1}$ for some edge $E$ of $\Gamma(f)$ and hence $Q \rightarrow Q_{E, 1}$. If $P$ is special, we may assume that $P=(0: 1)$. Then the local equation of the strict transform $\tilde{f}$ of $f$ at $P$ in chart 2 , is:

$$
\tilde{f}(u, v)=\frac{f(u v, v)}{v^{m}} .
$$

Since $n(\tilde{f}, Q)=n(f, Q)-1$ and by induction hypothesis, there is an edge $E^{\prime}$ of $\Gamma(\tilde{f})$ such that

$$
\tilde{f}_{E^{\prime}}=\text { monomial } \times \prod_{i=1}^{s}\left(a_{i} u^{m_{0}^{\prime}}-b_{i} v^{n_{0}^{\prime}}\right)^{r_{i}},
$$

where $a_{i}, b_{i} \in K^{*},\left(a_{i}: b_{i}\right)$ pairwise distinct; $m_{0}^{\prime}, n_{0}^{\prime}, r_{i} \in \mathbb{N}_{>0}, \operatorname{gcd}\left(m_{0}^{\prime}, n_{0}^{\prime}\right)=1$ and $Q \rightarrow Q_{E^{\prime}, i}$ for some $i$. Let $m_{0}=m_{0}^{\prime}, n_{0}=m_{0}^{\prime}+n_{0}^{\prime}$ and let $f=f_{d}^{\mathrm{w}}+f_{d+1}^{\mathrm{w}}+\ldots$ be the $\left(n_{0}, m_{0}\right)$-weighted homogeneous decomposition of $f$. Then for each $l>d$, we have

$$
\begin{aligned}
\frac{f_{l}^{\mathrm{w}}(u v, v)}{v^{m}} & =\sum_{n_{0} \alpha+m_{0} \beta=l} c_{\alpha \beta}(u v)^{\alpha} v^{\beta-m} \\
& =\sum_{n_{0}^{\prime} \alpha+m_{0}^{\prime}(\alpha+\beta-m)=l-m m_{0}^{\prime}} c_{\alpha \beta} u^{\alpha} v^{\alpha+\beta-m} .
\end{aligned}
$$

This implies that $\tilde{f}=\tilde{f}_{e}^{\mathrm{w}}+\tilde{f}_{e+1}^{\mathrm{w}}+\ldots$ is the $\left(n_{0}^{\prime}, m_{0}^{\prime}\right)$-weighted homogeneous decomposition of $\tilde{f}$, where $e=d-m m_{0}^{\prime}$ and $\tilde{f}_{l-m m_{0}^{\prime}}^{\mathrm{w}}=\frac{f_{l}(u v, v)}{v^{m}}$. Note that $\tilde{f}_{e}^{\mathrm{w}}=\tilde{f}_{E^{\prime}}$. It is easy to see that

$$
f_{d}^{\mathrm{w}}(x, y)=y^{m} \tilde{f}_{e}^{\mathrm{w}}\left(\frac{x}{y}, y\right)=\text { monomial } \times \prod_{i=1}^{s}\left(a_{i} x^{m_{0}}-b_{i} y^{n_{0}}\right)^{r_{i}} .
$$

Since $E^{\prime}$ is an edge of $\Gamma\left(\tilde{f}_{E^{\prime}}\right), \tilde{f}_{E^{\prime}}$ and then $f_{d}^{\mathrm{w}}(x, y)$ are not monomials. By $E$ we denote the convex hull of the support of $f_{d}^{\mathrm{w}}$. Then $E$ is an edge of $\Gamma(f)$ and $f_{E}=f_{d}^{\mathrm{w}}$. Therefore $Q_{E, i}=Q_{E^{\prime}, i}$ and hence $Q \rightarrow Q_{E, i}$. 
Proof of Theorem 3.12. ( $\Longrightarrow$ ): Assume $f$ is not WHNND, then $f$ is not WHND along some edge $E$ of $\Gamma(f)$. By Lemma 3.13, there is an infinitely near point $Q_{E, i}$ of 0 , such that $m_{Q_{E, i}}>1$. Clearly, $Q_{E, i}$ is not special. It then follows from Proposition 3.9 that

$$
\delta(f)>\nu(f)=\delta_{N}(f) .
$$

$(\Longleftarrow)$ : Assume now that $f$ is WHNND. To show $\delta(f)=\delta_{N}(f)$, it suffices to show that there is no infinitely near point $Q$ of 0 such that $Q$ is not special and $m_{Q}>1$. We argue by contradiction. Suppose that there is such an infinitely near point $Q$. By Lemma 3.15, there is an edge $E$ of $\Gamma(f)$ such that $Q \rightarrow Q_{E, i}$ for some $i$, and then $m_{Q} \leq m_{Q_{E, i}}$. Since $f$ is WHND along $E$, it follows from Lemma 3.14 that $m_{Q_{E, i}}=1$. Hence $m_{Q} \leq m_{Q_{E, i}}=1$, which is a contradiction.

If $\operatorname{char}(K)=0$ we have Milnor's famous formula $\mu(f)=2 \delta(f)-r(f)+1$, where $r(f)$ is the number of branches of $f$. The formula is wrong in general if $\operatorname{char}(K)>0$ but still holds if $f$ is NND by [BGM10, Thm. 4.13]. Using the general inequality

$$
\mu_{N}(f)=2 \delta_{N}(f)-r_{N}(f)+1 \leq 2 \delta(f)-r(f)+1 \leq \mu(f)
$$

from [BGM10], then Theorem 2.13, Proposition 3.1 and Proposition 3.10 imply

Corollary 3.16. Let $f \in K[[x, y]]$ be reduced. Then $f$ is INND if and only if $f$ is $W N N D$ and $\mu(f)=2 \delta(f)-r(f)+1$.

Remark 3.17. (1) The difference wvc $(f):=\mu(f)-2 \delta(f)+r(f)-1$ counts the number of wild vanishing cycles of (the Milnor fiber) of $f$ (cf. [Del73], [MHW01], [BGM10]), which vanishes if $\operatorname{char}(K)=0$ or if $f$ is INND.

(2) $\operatorname{wvc}(f)$ is computable for any given $f$. This follows since $\mu(f)$ is computable by a standard basis computation w.r.t. a local ordering (cf. [GP08]) and $\delta(f)$ and $r(f)$ are computable by computing a Hamburger-Noether expansion (cf. Cam80]). Both algorithms are implemented in Singular (cf. GPS05]).

Example 3.18. Consider $f=x(x-y)^{2}+y^{7}$ and $g=x(x-y)^{2}+y^{7}+x^{6}$ and char $(K)=3$. Using Singular we compute $\mu(f)=8, \delta(f)=5, r(f)=3$ and $\mu(g)=8, \delta(f)=4, r(g)=2$. We have $\operatorname{wvc}(f)=0, \operatorname{wvc}(g)=1, \Gamma(f)=\Gamma(g)$ and $f$ is not INND. This shows

- INND is sufficient but not necessary for the absence of wild vanishing cycles,

- the Newton diagram can not distinguish between singularities which have wild vanishing cycles and those which have not. 
Although we can compute the number of wild vanishing cycles, it seems hard to understand them. We like to pose the following

Problem. Is there any "geometric" way to understand the wild vanishing cycles, distinguishing them from the ordinary vanishing cycles counted by $2 \delta-r+1$ ? Is there at least a "reasonable" characterization of those singularities without wild vanishing cycles?

Acknowledgments. We would like to thank the referee for his comments and suggestions which prompted us to improve the paper.

\section{REFERENCES}

[BeP00] P. Beelen and R. Pellikaan, The Newton polygon of plane curves with many rational points, Designs, Codes and Cryptography 21(2000), 41-67.

[BGM10] Y. Boubakri, G.-M. Greuel, and T. Markwig, Invariants of Hypersurface Singularities in Positive Charecteristic, Rev. Mat. Complut. (2010) DOI 10.1007/s13163-010-0056-1, 23 pages.

[Biv09] C. Bivià-Ausina, Local Eojasiewicz exponents, Milnor numbers and mixed multiplicities of ideals, Math. Z. 262(2009), no. 2, 389-409.

[BrK86] E. Brieskorn; H. Knörrer, Plane Algebraic Curves, Birkhaeuser (1986), 721 pages.

[Cam80] A. Campillo, Algebroid Curves in Positive Characteristic, Lecture Notes in Math. vol. 613, Springer-Verlag (1980), 168 pages.

[Del73] P. Deligne, La formule de Milnor, Sém. Géom. Algébrique du Bois-Marie, 1967-1969, SGA 7 II, Lecture Notes in Math. 340, Expose XVI, 197-211 (1973), 1973.

[GP08] G.-M. Greuel, G. Pfister, A Singular Introduction to Commutative Algebra, 2nd Edition, Springer (2008), 702 pages.

[GPS05] G.-M. Greuel, G. Pfister, H. Schönemann, Singular 3.1.0, A Computer Algebra System for Polynomial Computations. Centre for Computer Algebra, University of Kaiserslautern (2005). http://www.singular.uni-kl.de.

[GLS06] G.-M. Greuel, C. Lossen, and E. Shustin, Introduction to Singularities and Deformations, Math. Monographs, Springer-Verlag (2006), 476 pages.

[Kou76] A. G. Kouchnirenko, Polyèdres de Newton et nombres de Milnor, Invent. Math. 32 (1976), 1-31.

[Lu87] I. Luengo, The $\mu$-constant stratum is not smooth, Invent. Math., 90 (1987), 139-152.

[MHW01] A. Melle-Hernández and C. T. C. Wall, Pencils of curves on smooth surfaces, Proc. Lond. Math. Soc., III. Ser. 83 (2001), no. 2, 257-278.

[Wal99] C. T. C. Wall, Newton polytopes and non-degeneracy, J. reine angew. Math. 509 (1999), 1-19. 
Gert-Martin Greuel

Universität Kaiserslautern, Fachbereich Mathematik, Erwin-Schrödinger-Strasse, 67663 Kaiserslautern, Tel. +496312052850, Fax +496312054795

E-mail address: greuel@mathematik.uni-kl.de

NGUYEN HONG Dud3

Institute of Mathematics, 18 HoAng Quoc Viet Road, Cau Giay District 10307, HanoI.

E-mail address: nhduc@math.ac.vn

Universität Kaiserslautern, Fachbereich Mathematik, Erwin-Schrödinger-Strasse, 67663 KAISERSLAUTERN

E-mail address: dnguyen@mathematik.uni-kl.de

\footnotetext{
${ }^{3}$ Supported by DAAD (Germany) and NAFOSTED (Vietnam)
} 